\title{
New Proposals on Portuguese Vegetation (II)
}

\author{
Carlos Pinto-Gomes, Rodrigo Paiva-Ferreira \& Catarina Meireles (*)
}

\begin{abstract}
Pinto-Gomes, C., Paiva-Ferreira, R. \& Meireles, C. New Proposals on Portuguese Vegetation (II). Lazaroa 31 : 59-65 (2010).

As a consequence of the geobotanical studies that have been developed in west and southern areas of the Iberian Peninsula, four new phytosociological associations are proposed, integrated in the perennial mesophytic grasslands of Stipo giganteae-Agrostietea castellanae (Serratulo flavescentis-Celticetum giganteae, Armerio macrophyllae-Celticetum giganteae, Centaureo rothmaleranae-Celticetum giganteae, and Centaureo coutinhoi-Dactyletum lusitanici). For each proposed unit, besides its syntaxonomical fitting, we present its ecological diagnosis and the serial and catenal context, as well as its patrimonial value.
\end{abstract}

Key-words: Phytosociology, Iberian Peninsula, perennial grasslands.

Resumo: Pinto-Gomes, C., Paiva-Ferreira, R. \& Meireles, C. Novas propostas sobre a vegetação portuguesa (II). Lazaroa 31: 59-65 (2010).

Em consequência dos estudos geobotânicos que se têm vindo a desenvolver no oeste e sul da Península Ibérica, propõem-se quatro novas associações vegetais no âmbito dos arrelvados vivazes mesofíticos da Stipo giganteae-Agrostietea castellanae (Serratulo flavescentis-Celticetum giganteae, Armerio macrophyllae-Celticetum giganteae, Centaureo rothmaleranae-Celticetum giganteae e Centaureo coutinhoi-Dactyletum lusitanici). Para cada unidade proposta, para além do seu enquadramento sintaxonómico, apresenta-se a respectiva diagnose ecológica e o contexto serial e catenal, bem como o valor patrimonial que encerra.

Palavras chave: Fitossociologia, Península Ibérica, arrelvados vivazes.

\section{INTRODUCTION}

Continuing the studies developed by the University of Évora Geobotanical Group, several plant communities of high originality are presented. Thus, as a corollary, we present four new herbaceous perennials associations that integrate the dynamics of cork oak, holm oaks and oaks, distributed in central and southern Portugal. In methodological terms, the plant material was identified using preferably CASTROVIEJO \& al.(1986-2005), COUTINHO (1939), Franco $(1971,1984)$, Franco \& AFONSO (1994, 1999), SAMPAIO (1988), Tutin et al. (19641980), VALDES et al. (1987) and specifically for
Celtica VÁZquEZ \& BARKWORTH (2004). In terms phytosociological nomenclature the method developed by BRAUN-BLANQUET (1965) and GÉHU \& RIVAS-MARTínEZ (1981) was followed.

\section{PHYTOSOCIOLOGICAL ANALYSIS}

Serratulo flavescentis-Celticetum giganteae C. Pinto-Gomes \& R. Paiva-Ferreira ass. nova hoc loco (Table 1, holotypus rel. 4).

Diagnosis. Cespititious oceanic thermomediterranean dry to subhumid perennial grasslands, over deep limy substrata (typical of dolomitic

\footnotetext{
* Departament of Paisagem, Ambiente e Ordenamento (DPAO/ICAAM). University of Évora. Rua Romão Ramalho 59. 7002-671 Évora. Portugal. E-mail: cpgomes@uevora.pt.
} 
Table 1

Serratulo flavescentis-Celticetum giganteae C. Pinto-Gomes \& R. Paiva-Ferreira ass. nova hoc loco (Agrostio castellanae-Stipion giganteae, Agrostietalia castellanae, Stipo giganteae-Agrostietea castellanae)

\begin{tabular}{|c|c|c|c|c|c|c|c|}
\hline Altitude (m.a.s.1.) & 20 & 18 & 34 & 25 & 21 & 29 & \\
\hline Area (sq.m) & 100 & 100 & 100 & 100 & 100 & 100 & \\
\hline Cover $(\%)$ & 80 & 80 & 70 & 70 & 70 & 70 & \\
\hline Exposure & - & - & S & S & S & S & \\
\hline Slope (o) & - & - & 4 & 2 & 2 & 2 & \\
\hline Vegetation height (m) & 1,5 & 1,5 & 1,5 & 1,5 & 1,5 & 1,5 & \\
\hline Number of taxa & 24 & 23 & 19 & 19 & 15 & 16 & \\
\hline Number & 1 & 2 & 3 & 4 & 5 & 6 & 7 \\
\hline \multicolumn{8}{|l|}{ Characteristic taxa } \\
\hline Celtica gigantea & 4 & 4 & 3 & 4 & 3 & 3 & $\mathrm{~V}$ \\
\hline Bupleurum rigidum subsp. paniculatum & 2 & 2 & 1 & 2 & 1 & + & $\mathrm{V}$ \\
\hline Arrhenatherum album & 1 & + & + & 2 & 2 & + & $\mathrm{V}$ \\
\hline Serratula flavescens & 1 & 1 & + & 1 & 2 & 2 & $\mathrm{~V}$ \\
\hline Dactylis hispanica & 2 & 2 & 1 & 1 & . & + & $\mathrm{V}$ \\
\hline Thapsia villosa & . & 1 & 1 & 1 & + & 2 & IV \\
\hline Asphodelus aestivus & . & . & . & 1 & + & + & II \\
\hline \multicolumn{8}{|l|}{ Other taxa } \\
\hline Carex halleriana & + & + & 1 & + & + & 1 & $\mathrm{~V}$ \\
\hline Chamaerops humilis & + & + & 1 & + & + & 1 & $\mathrm{~V}$ \\
\hline Phlomis lycnhitis & + & + & 1 & + & + & + & $\mathrm{V}$ \\
\hline Gladiolus illyricus & + & + & . & 1 & + & 1 & IV \\
\hline Brachypodium phoenicoides & + & 1 & . & 1 & + & 2 & IV \\
\hline Eryngium dilatatum & 1 & 1 & . & + & . & 2 & III \\
\hline Allium roseum & . & . & + & + & + & + & III \\
\hline Phlomis purpurea & + & + & 1 & . & . & . & II \\
\hline Sanguisorba minor subsp. spachiana & + & 1 & . & . & . & . & II \\
\hline Melica minuta & 1 & 1 & . & . & . & . & II \\
\hline Galium concatenatum & + & & + & & . & . & II \\
\hline Genista algarbiensis & + & . & + & . & . & . & II \\
\hline Urginea maritima & + & . & + & . & . & . & II \\
\hline Asperula hirsuta & 1 & . & . & + & . & + & II \\
\hline Rhamnus oleoides & + & . & . & + & . & + & II \\
\hline Clematis flammula & + & . & . & + & . & . & II \\
\hline Teucrium pseudochamaepitys & + & . & . & + & . & . & II \\
\hline Salvia sclareoides & . & + & + & . & . & . & II \\
\hline
\end{tabular}

Other taxa: Macrochloa tenacissima + in 1 and 5; Aristolochia baetica and Carlina corymbosa + in 1; Ulex argenteus, Phillyrea angustifolia, Arbutus unedo, Cistus monspeliensis and Ceratonia siliqua + , Centaurium erythraea and Iris xiphium 1 in 2; Plantago lanceolata, Ornithogalum narbonense, Atractylis gummifera and Asphodelus ramosus + in 3; Scilla peruviana + in 4 .

Localities: 1: Cerro da Cabeça; 2: Tavira; 3: Loulé; 4: Between Faro and Olhão (near Via do Infante), holotypus ass.; 5: near Fonte Benémola; 6: Salir; 7: synthetic relevé.

"terra rossa" soils more or less profound), exclusive of the Algarvian sector. In the list of flora, some Portuguese endemic characteristic species of these southern territories can be noted, such as Serratula flavescens, as well as the presence of several Stipo giganteae-Agrostietea castellanae taxa (Arrhenatherum album, Dactylis hispanica,
Bupleurum rigidum subsp. paniculatum and Thapsia villosa). This association is geovicariant of Avenulo occidentalis-Celticetum giganteae $\mathrm{R}$. Paiva-Ferreira in Pinto-Gomes, Paiva-Ferreira \& Meireles 2007 (this last one exclusive to the Ribatagan-Sadensean and Dividing Portuguese sectors). 
Serial considerations. Lategraminetum of Querco alpestris-broteroi S. and Rhamno oleioidis-Querco rotundifoliae $S$. This association is frequently in mosaic with sclerophytic brushwoods: Asparago albi-Rhamnion oleoidis Rivas Goday ex Rivas-Martínez 1975, Siderito lusitanicae-Genistetum algarbiensis Pinto-Gomes \& Paiva-Ferreira 2005 and Galio concatenatiBrachypodietum phoenicoidis Pinto-Gomes \& Paiva-Ferreira 2005.

Armerio macrophyllae-Celticetum giganteae $\mathrm{R}$. Paiva-Ferreira, C. Pinto-Gomes \& R. Pinto ass. nova hoc loco (Table 2, holotypus rel. 4).

Diagnosis. Cespititious oceanic thermomediterranean perennial grasslands, dry to subhumid, typical of pliopleistocene sandy soils more or less profound, exclusive of the Algarvean sector. In the list of flora, some Portuguese endemic characteristic species of these southern territories can be noted, such as Armeria macrophylla, as well as the presence of several Stipo giganteae-Agrostietea castellanae taxa (Arrhenatherum album, Dactylis hispanica subsp. lusitanica, Asphodelus aestivus, Thapsia villosa, Margotia gummifera and Scilla odorata).

Seral considerations. Lategraminetum of Oleo sylvestris-Querco suberis $S$. This association is frequently found in contact with sclerophytic brushwoods of Coremation albi Rothmaler 1943, Ericion umbellatae Br.-B1., P.Silva, Rozeira \& Fontes 1952, Stauracanthion boivinii (Rivas-Martínez 1979) Rivas-Martínez, Fernández-González \& Loidi 1999 and Pistacio lentisci-Rhamnetalia alaterni Rivas-Martínez 1975 formations.

\section{Centaureo rothmaleranae-Celticetum giganteae}

C. Meireles \& C. Pinto-Gomes ass. nova hoc loco (Table 3, holotypus rel. 6).

Diagnosis. Siliceous perennial grasslands dominated by Celtica gigantea, typical of deep soils with an organic horizon well defined, exclusive of estrelensean supramediterranean territories and surrounding areas with submediterranean influence. It's an association with some similarity to Arrhenatheruo baetici-Stipetum giganteae Rivas-Martínez, Fernández-González \& SánchezMata 1986, described for supramediterranean
Carpetanean-leonesean territories, differing significantly from the ecological and floristic points of view. One of the most important differences is the absence of Arrhenatherum elatius subsp. baeticum, Thymus bracteatus and Armeria arenaria subsp. segoviensis, the last two endemics from Spanish territories. On the other hand, Centaureo rothmaleranae-Celticetum giganteae has plants like Arrhenatherum elatius subsp. carpetanum, Agrostis x fouilladei and Centaurea rothmalerana, this last one endemic from Serra da Estrela.

Serial considerations. Lategraminetum of Holco mollis-Quercetum pyrenaicae $S$. This association is frequently found in contact with several communities like Cytiso striati-Genistetum polygaliphyllae Rivas-Martínez 1981 or Phalacrocarpo oppositifolii-Festucetum elegantis Rivas-Martínez, T.E. Díaz, F. Prieto, Loidi \& Penas in E. Puente 1988.

\section{Centaureo coutinhoi-Dactyletum lusitanici $\mathrm{C}$.} Meireles \& C. Pinto-Gomes ass. nova hoc loco (Table 4, holotypus rel. 6).

Diagnosis. Mesomediterranean siliceous perennial grassland, typical of deep and well structured substrata (but not hydromorfic), that occurs in the meridional Carpetanean and septentrional Luso-extremadurensean territories, always in strong thermophilic and mediterranean influenced areas. It is a low dense formation, dominated by Dactylis hispanica subsp. lusitanica and Centaurea coutinhoi. Can be distinguished from SedoAgrostietum castellanae by the presence and dominance of Dactylis hispanica subsp. lusitanica, and termophilic taxa like Centaurea coutinhoi and Pimpinella villosa, among others. Although this new association has Agrostis castellana, it is very different from Gaudinio fragilis-Agrostietum castellanae since it does not live in places with hydromorphism, even temporarily.

Serial considerations. Subserial grassland of Arisaro-Querco pyrenaicae and Arbuto unedonisQuerco pyrenaicae forests. In dry to subhumid territories is included in Sanguisorbo hybridaeQuerco suberis $S$. and Pyro bourgaeanae-Querco rotundifoliae $S$. This association is frequently found in contact with Cytiso multiflori-Sarothamnetum eriocarpi Rivas Goday 1964, Polygalo microphyllae-Cistetum populifolii Rivas Goday 1964, Melico 
Table 2

Armerio macrophyllae-Celticetum giganteae R. Paiva-Ferreira, C. Pinto-Gomes \& R. Pinto ass. nova hoc loco (Agrostio castellanae-Stipion giganteae, Agrostietalia castellanae, Stipo giganteae-Agrostietea castellanae)

\begin{tabular}{|c|c|c|c|c|c|c|c|c|}
\hline Altitude (m.a.s.1.) & 20 & 20 & 10 & 10 & 10 & 15 & 10 & \\
\hline Area (sq.m) & 50 & 50 & 100 & 150 & 60 & 200 & 100 & \\
\hline Cover $(\%)$ & 80 & 65 & 70 & 90 & 70 & 80 & 70 & \\
\hline Orientation & - & - & - & - & - & - & - & \\
\hline Slope (o) & 0 & 0 & 0 & 0 & 0 & 0 & 0 & \\
\hline Vegetation height (m) & 1,5 & 1,5 & 1,5 & 1,5 & 1,5 & 2 & 1,5 & \\
\hline Number of taxa & 16 & 15 & 18 & 25 & 19 & 27 & 25 & \\
\hline Number & 1 & 2 & 3 & 4 & 5 & 6 & 7 & 8 \\
\hline
\end{tabular}

Characteristic taxa

Celtica gigantea

Armeria macrophylla

Asphodelus aestivus

Thapsia villosa

Scilla odorata

Arrhenatherum album

Margotia gummifera

Serratula monardii

Other taxa

Cytisus grandiflorus subsp. cabezudoi

Chamaerops humilis

Cistus libanotis

Lavandula sampaioana subsp lusitanica

Halimium calycinum

Iris xiphium

Thymus albicans

Stauracanthus genistoides

Halimium halimifolium

Urginea maritima

Asparagus aphyllus

Thymus lotocephalus

Ulex argenteus subsp. subsericeus

Iberis ciliata subsp. welwitschii

Dianthus broteri subsp. hinoxianus

Gladiolus illyricus

Aristolochia baetica

Pycnocomon rutifolium

Ulex australis subsp. welwitschianus

Dittrichia viscosa subsp. revoluta

Centaurea aspera subsp. stenophylla

Corynephorus canescens var. maritimus

Malcolmia lacera subsp. gracilima

Tuberaria major

Arisarum vulgare

Euphorbia baetica

Rhamnus oleoides

Scilla monophyllus

\begin{tabular}{|c|c|c|c|c|c|c|c|}
\hline 4 & 4 & 4 & 5 & 4 & 4 & 4 & V \\
\hline 2 & 2 & 2 & 2 & 2 & 1 & 2 & $\mathrm{~V}$ \\
\hline 1 & 2 & + & 1 & 2 & 3 & 2 & V \\
\hline 1 & 1 & 1 & 1 & 2 & 1 & 2 & V \\
\hline 1 & 2 & 1 & 1 & . & 1 & + & V \\
\hline . & + & 1 & 2 & 2 & 2 & + & V \\
\hline 1 & . & . & . & + & 1 & 2 & III \\
\hline$\cdot$ & . & . & . & . & . & 1 & I \\
\hline+ & + & . & + & + & + & + & V \\
\hline+ & + & + & . & . & + & + & IV \\
\hline+ & . & . & + & + & + & + & IV \\
\hline+ & . & . & + & + & + & + & IV \\
\hline+ & . & + & + & . & 1 & + & IV \\
\hline$\cdot$ & . & 1 & 1 & 1 & 1 & 1 & IV \\
\hline+ & + & . & . & + & + & . & III \\
\hline+ & + & . & . & . & + & + & III \\
\hline$\cdot$ & . & + & + & + & + & . & III \\
\hline . & . & + & + & . & 1 & + & III \\
\hline$\cdot$ & + & . & . & . & + & + & III \\
\hline . & + & . & 1 & . & . & 2 & III \\
\hline . & . & + & 1 & + & . & . & III \\
\hline+ & . & . & . & . & + & . & II \\
\hline$\cdot$ & 1 & . & . & . & 2 & . & II \\
\hline$\cdot$ & . & . & . & 1 & . & + & II \\
\hline$\cdot$ & . & + & + & . & . & . & II \\
\hline$\cdot$ & . & + & + & . & . & . & II \\
\hline$\cdot$ & . & . & . & . & + & + & II \\
\hline . & . & . & + & . & + & . & II \\
\hline 1 & . & . & . & + & . & . & II \\
\hline+ & . & . & . & . & . & + & II \\
\hline$\cdot$ & + & . & . & . & + & . & II \\
\hline . & . & . & . & 1 & . & + & II \\
\hline$\cdot$ & $\cdot$ & 1 & + & . & . & . & II \\
\hline . & . & . & 1 & + & . & . & II \\
\hline$\cdot$ & $\cdot$ & . & + & . & + & . & II \\
\hline . & . & . & . & . & + & + & II \\
\hline
\end{tabular}

Other taxa: Aetheorhiza bulbosa and Rubia peregrina subsp. longifolia + in 3; Carlina corymbosa, Corrigiola litoralis and Orobanche gracilis + in 4; Ruta chalepensis + in 5; Pulicaria odora + in 6; Hyparrhenia hirta subsp. pubescens + in 7.

Localities: 1: Açoteias (Albufeira); 2: Pinhal de Belmonte (Olhão); 3: Quinta de Marim (Olhão); 4: Parque Natural da Ria Formosa Burreau (Olhão), holotypus ass.; 5: Ludo (Faro); 6: Pontal (Faro); 7: Gambelas (Faro); 8: synthetic relevé. 
Table 3

Centaureo rothmaleranae-Celticetum giganteae C. Meireles \& C. Pinto-Gomes ass. nova hoc loco (Festucion merinoi, Agrostietalia castellanae, Stipo giganteae-Agrostietea castellanae)

\begin{tabular}{|c|c|c|c|c|c|c|c|}
\hline Altitude (1=10 m.) & 110 & 137 & 118 & 95 & 120 & 104 & \\
\hline Area (sq.m) & 400 & 300 & 300 & 150 & 100 & 400 & \\
\hline Cover $(\%)$ & 95 & 90 & 95 & 85 & 90 & 95 & \\
\hline Orientation & $\mathrm{O}$ & $\mathrm{S}$ & $\mathrm{E}$ & $\mathrm{O}$ & $\mathrm{E}$ & $\mathrm{E}$ & \\
\hline Slope (o) & 25 & 18 & 20 & 30 & 30 & 20 & \\
\hline Vegetation height (m) & 1,5 & 1,5 & 1,5 & 1,5 & 1,5 & 1,5 & \\
\hline Number of taxa & 19 & 19 & 14 & 19 & 20 & 26 & \\
\hline Number & 1 & 2 & 3 & 4 & 5 & 6 & 7 \\
\hline \multicolumn{8}{|l|}{ Characteristic taxa } \\
\hline Celtica gigantea & 4 & 4 & 5 & 4 & 4 & 4 & $\mathrm{~V}$ \\
\hline Centaurea rothmalerana & 2 & 1 & 1 & 1 & 1 & 2 & $\mathrm{~V}$ \\
\hline Agrostis $\mathrm{x}$ fouilladei & 2 & 1 & 2 & 1 & . & 2 & $\mathrm{~V}$ \\
\hline Arrhenatherum carpetanum & 2 & 1 & 1 & 2 & . & 2 & $\mathrm{~V}$ \\
\hline Rumex acetosella subsp. angiocarpus & . & . & + & 1 & . & 1 & III \\
\hline Festuca elegans subsp. merinoi & . & 2 & . & . & 2 & . & II \\
\hline Sanguisorba verrucosa & . & . & . & + & + & . & II \\
\hline Armeria beirana & + & . & . & 1 & . & . & II \\
\hline Malva tournefortiana & . & . & . & . & + & . & I \\
\hline Avenula sulcata & . & + & . & . & . & . & I \\
\hline $\begin{array}{l}\text { Dactylis glomerata subsp. lusitanica } \\
\text { Other taxa }\end{array}$ & . & . & . & . & . & + & I \\
\hline Hypericum linarifolium & 1 & . & + & + & 1 & + & $\mathrm{V}$ \\
\hline Agrostis truncatula & 1 & + & 1 & + & . & 2 & $\mathrm{~V}$ \\
\hline Sesamoides suffruticosa & 1 & . & + & + & . & 1 & $\mathrm{~V}$ \\
\hline Hypochoeris radicata & + & . & . & + & + & 1 & IV \\
\hline Jasione sessiliflora & 1 & + & . & . & + & 1 & IV \\
\hline Deschampsia flexuosa subsp. iberica & + & 1 & . & + & . & + & IV \\
\hline Halimium alyssoides & . & 1 & . & . & 2 & + & III \\
\hline Lotus corniculatus subsp. carpetanus & + & + & . & . & + & . & III \\
\hline Allium sphaerocephalon & + & + & . & . & . & + & III \\
\hline Erica australis subsp. aragonensis & . & 1 & + & 2 & . & . & III \\
\hline Digitalis purpurea subsp. carpetana & . & . & + & + & . & 1 & III \\
\hline Jasione montana & . & . & 1 & + & . & + & III \\
\hline Viola langeana & . & + & + & . & . & + & III \\
\hline Coincya monensis subsp. orophila & + & . & . & . & + & . & II \\
\hline Arenaria montana & . & + & . & . & 2 & . & II \\
\hline Cytisus multiflorus & . & . & . & 2 & 1 & . & II \\
\hline Clinopodium vulgare & + & . & . & + & . & . & II \\
\hline Dianthus lusitanicus & . & + & . & + & . & . & II \\
\hline Corynephorus canescens var. montana & . & + & . & . & . & + & II \\
\hline Genista florida subsp. polygaliphylla & . & . & + & . & . & + & II \\
\hline Poa bulbosa & + & . & . & . & . & + & II \\
\hline Phalacrocarpon oppositifolium & . & + & + & . & . & . & II \\
\hline
\end{tabular}

Other taxa: Festuca summilusitana 1, Digitalis thapsi and Silene nutans + in 1; Luzula lactea + in 2; Lactuca serriola + in 4; Erica arborea 1, Anarrhinum bellidifolium, Cytisus striatus, Lactuca viminea, Linaria saxatilis, Quercus pyrenaica and Sedum hirsutum + in 5; Pteridium aquilinum 1, Carduus carpetanus, Festuca ampla, Holcus gayanus, Silene latifolia and Teucrium salviastrum + in 6.

Localities: 1: Albercães; 2: Albergaria; 3: Vale Mourisco; 4: Manteigas: S. Pedro, near Lameiras; 5: Manteigas: near Carvalheira; 6: Albercães, holotypus ass.; 7: synthetic relevé. 
Table 4

Centaureo coutinhoi-Dactyletum lusitanici C. Meireles \& C. Pinto-Gomes ass. nova hoc loco (Agrostion castellanae, Agrostietalia castellanae, Stipo giganteae-Agrostietea castellanae)

\begin{tabular}{|c|c|c|c|c|c|c|c|}
\hline Altitude (m.a.s.1.) & 530 & 630 & 550 & 540 & 650 & 280 & \\
\hline Area (sq.m) & 25 & 2 & 15 & 16 & 20 & 20 & \\
\hline Cover $(\%)$ & 90 & 80 & 85 & 90 & 98 & 80 & \\
\hline Orientation & S & SE & $\mathrm{SO}$ & S & $\mathrm{N}$ & SE & \\
\hline Slope (o) & 25 & 0 & 5 & 8 & 20 & 20 & \\
\hline Vegetation height (m) & 30 & 30 & 30 & 30 & 30 & 30 & \\
\hline Number of taxa & 17 & 12 & 19 & 18 & 15 & 20 & \\
\hline Number & 1 & 2 & 3 & 4 & 5 & 6 & 7 \\
\hline \multicolumn{8}{|l|}{ Characteristic taxa } \\
\hline Dactylis hispanica subsp. lusitanica & 5 & 2 & 4 & 5 & 4 & 4 & $\mathrm{~V}$ \\
\hline Centaurea coutinhoi & 2 & 4 & 2 & 2 & . & 2 & V \\
\hline Agrostis x fouilladei & 1 & 2 & 2 & 1 & 3 & . & $\mathrm{V}$ \\
\hline Malva tournefortiana & . & 1 & 1 & . & . & 1 & III \\
\hline Agrostis castellana & . & . & 1 & 1 & . & 1 & III \\
\hline Sanguisorba verrucosa & . & . & 2 & + & . & 2 & III \\
\hline Euphorbia oxyphylla & . & . & + & + & + & . & III \\
\hline Thapsia minor & . & . & . & + & 1 & + & II \\
\hline Thapsia villosa & . & . & . & . & . & + & I \\
\hline Centaurea ornata & . & . & . & . & . & 1 & I \\
\hline Asphodelus aestivus & . & . & . & . & . & 1 & I \\
\hline Rumex acetosella subsp. angiocarpus & . & 1 & . & . & . & . & I \\
\hline \multicolumn{8}{|l|}{ Other taxa } \\
\hline Daucus carota & 1 & . & + & 2 & + & + & V \\
\hline Lactuca viminea & + & . & + & + & + & + & V \\
\hline Hypochoeris radicata & + & + & + & . & + & + & $\mathrm{V}$ \\
\hline Cistus ladanifer & + & 1 & . & . & 1 & + & IV \\
\hline Lotus corniculatus subsp. carpetanus & . & . & 1 & 1 & 2 & . & IV \\
\hline Cytisus multiflorus & 1 & . & . & . & + & + & III \\
\hline Pimpinella villosa & + & . & . & 2 & . & + & III \\
\hline Lavandula sampaioana & . & . & . & 1 & 1 & + & III \\
\hline Anarrhinum bellidifolium & . & + & . & . & + & + & III \\
\hline Lithodora prostrata & . & . & + & + & + & . & III \\
\hline Hypericum perforatum & + & . & + & . & . & + & III \\
\hline Melica ciliata subsp. magnolii & 1 & . & . & + & . & . & II \\
\hline Plantago lanceolata & + & 2 & . & . & . & . & II \\
\hline Helichrysum stoechas & . & . & . & 1 & 1 & . & II \\
\hline Pteridium aquilinum & . & . & + & 2 & . & . & II \\
\hline
\end{tabular}

Other taxa: Quercus rotundifoliae + in 4 and 6; Armeria beirana, Crataegus monogyna and Lactuca virosa 1, Lepidium heterophyllum and Rumex induratus + in 1; Cistus inflatus and Rumex acetosa 1, Arrhenatherum elatius subsp. bulbosum + in 2; Quercus pyrenaica 1, Aristolochia paucinervis, Clinopodium vulgare, Daphne gnidium and Fraxinus angustifolia + in 3; Lonicera periclymenum subsp. hispanica + in 4; Hypericum linarifolium + in 5; Quercus suber and Allium sphaerocephalon + in 6.

Localities: 1: Guarda: Valhelhas, S. Miguel; 2: Manteigas: Sameiro, near the village; 3: Manteigas: Vale de Amoreira, near Quinta do Cabecinho; 4: Guarda: Valhelhas, Serra Rachada; 5: Manteigas: Vale de Amoreira, near the village; 6: Idanha-a-Nova, Proênça-a-Velha, Malhadas Velhas, holotypus ass.; 7: synthetic relevé.

magnolii-Celticetum giganteae Rivas-Martínez ex Peinado \& Martínez-Parras 1985, Gaudinio fragilis-Agrostietum castellanae Rivas-Martínez \& Belmonte 1986, Triseto ovati-Agrostietum truncatulae
Rivas Goday 1958, Pterosparto lasianthi-Ericetum aragonensis Rothmaler 1954 em. Rivas-Martínez 1979 and Festuco amplae-Cynosuretum cristati Rivas-Martínez ex Fuente 1986. 


\section{CONCLUDING REMARKS}

Four new associations are proposed as a contribution to the knowledge of Stipo giganteae-Agros- tietea castellanae grasslands. All of them are rich in endemic plants and integrate the 6220 pseudo-steppe with grasses and annuals of the Thero-Brachypodietea (Annex I, Directive 92/43/EEC) priority habitat.

\section{SYNTAXONOMICAL SCHEME}

STIPO GIGANTEAE-AGROSTIETEA CASTELLANAE Rivas-Martínez, Fernández-González \& Loidi 1999. Agrostietalia castellanae Rivas Goday in Rivas-Martínez, Costa, Castroviejo \& E. Valdés 1980 Agrostio castellanae-Stipion giganteae Rivas Goday ex Rivas-Martínez \& Fernández-González 1991 Serratulo flavescentis-Celticetum giganteae C. Pinto-Gomes \& R. Paiva-Ferreira ass. nova Armerio macrophyllae-Celticetum giganteae R. Paiva-Ferreira, C. Pinto-Gomes \& R. Pinto ass. nova Centaureo coutinhoi-Dactyletum lusitanici C. Meireles \& C. Pinto-Gomes ass. nova

Festucion merinoi Rivas-Martínez \& Sánchez-Mata in Rivas-Martínez, Sánchez-Mata \& Fernández-González 1986 corr. Rivas-Martínez \& Sánchez-Mata 2002

Centaureo rothmaleranae-Celticetum giganteae C. Meireles \& C. Pinto-Gomes ass. nova

\section{REFERENCES}

Braun-Blanquet, J. - 1965 - Plant Sociology: the study of plant communities - Hafner, Londres, 439 p.

Castroviejo, S. \& als. (Eds.) -1986-2005- Flora Ibérica: Plantas Vasculares de la Península Ibérica e Islas Baleares, vols. 1-7, 9, 10, 14, 21 - Real Jardín Botánico, CSIC., Madrid.

Coutinho, A.X. - 1939 - Flora de Portugal (Plantas Vasculares), $2^{\mathrm{a}}$ ed. - Ed. Bertrand, Lisboa. 933 pp.

Franco J.A. - 1971-1984 - Nova Flora de Portugal, vols. I \& II - Sociedade Astória, Lda., Lisboa.

Franco J.A. \& Afonso M.L.R. - 1994-1998 - Nova Flora de Portugal, vol. III, Fasc. I. \& II - Escolar Editora, Lisboa.

Recibido: 4 noviembre 2009

Aceptado: 11 enero 2010
Gehú,J.M. \& Rivas-Matínez,S. - 1981 - Notions Fondamentales de Phytossociologie in Syntaxonomie - J.Cramer, Vaduz, p.6-13.

Sampaio, G. - 1986- Flora Portuguesa ( $3^{\text {a }}$ ed.) - Ed. Imprensa Portuguesa, Porto.

Tutin, T.G., Heywood, V.H., Burges, N.A., Moore D. M., Valentina, D.H., Walters, S.M. \& Webb,D.A.(Eds.) - 1964-1980 - Flora Europaea, vols. I-IV - Cambridge University Press, Cambridge. Valdés, B., Talavera, S. \& Fernández-Galiano, E. -1987Flora Vascular de Andalusia Occidental, vols. 1-3 Ketres Editora S. A., Barcelona.

Vázquez, F. M. \& Barkworth, M.E. -2004- Resurrection and Emendation of Macrochloa (Gramineae: Stipeae) — Bot. J. Linn. Soc. 144 (4): 483-495. 